\title{
z Sonatem \\ Remifentanil Target Controlled Infusion for conscious sedation in a pregnant woman: safety and efficacy
}

Camilla Munafo'

San Camillo Forlanini Hospital: Azienda Ospedaliera San Camillo Forlanini

Fulvio Mammarella

San Camillo Forlanini Hospital: Azienda Ospedaliera San Camillo Forlanini

Antonella Loperfido ( $\nabla$ antonella_lop@hotmail.it )

Azienda Ospedaliera San Camillo Forlanini https://orcid.org/0000-0002-7174-8126

Arianna Crosti

Sapienza University of Rome: Universita degli Studi di Roma La Sapienza

Federico lannilli

Sapienza University of Rome: Universita degli Studi di Roma La Sapienza

Stefano Millarelli

San Camillo Forlanini Hospital: Azienda Ospedaliera San Camillo Forlanini

Gianluca Bellocchi

San Camillo Forlanini Hospital: Azienda Ospedaliera San Camillo Forlanini

Luigi Tritapepe

San Camillo Forlanini Hospital: Azienda Ospedaliera San Camillo Forlanini

\section{Case Study}

Keywords: Conscious Sedation, Analgosedation, Remifentanil, Pregnancy, Functional Endoscopic Sinus Surgery, FESS

Posted Date: August 17th, 2021

DOl: https://doi.org/10.21203/rs.3.rs-783467/v1

License: (c) (i) This work is licensed under a Creative Commons Attribution 4.0 International License. Read Full License 


\section{Abstract}

Background: Every year $2 \%$ of pregnant women undergo non-obstetric surgical interventions worldwide. According to the American College of Obstetricians and Gynaecologists Committee on Obstetric Practice, pregnant women should never be denied the most appropriate surgical treatment regardless of the trimester of pregnancy.

However, additional attention should be paid during the first trimester since there is the highest risk of inducing teratogenic mutations; similarly, during the third trimester, due to the possibility of preterm birth and low weight of the new-born great care should be paid.

Case presentation: We present the case of a 36-year-old woman during her $21^{\text {st }}$ week of pregnancy, with a normal-sized foetus according to the gestational age on ultrasound exam and with no additional risk factors. The patient referred an increasing nasal obstruction associated with rhinorrhoea of the left nasal cavity. She also reported episodes of sleep apnoea and hyposmia.

The patient received a detailed otolaryngological examination which allowed to identify a mass within the left nasal cavity. The subsequent nasal endoscopy confirmed a greyish polypoid mass lesion with a multinodular surface occupying the entire left nasal fossa. The lesion totally obliterated the left maxillary sinus resulting in obstruction of the anterior osteo-meatal unit and ethmoidal sinusitis She was referred for functional endoscopic sinus surgery (FESS) using analgosedation with Remifentanil Target Controlled Infusion.

Discussion and conclusions: To the very best of our knowledge this is the first case described in English literature about the use of analgosedation with Remifentanil Target Controlled Infusion for otolaryngology surgery, specifically in FESS. It could be an interesting option to avoid the use of inhaled anaesthetics that could induce foetal damage, especially during the first months of pregnancy.

Furthermore, patient intubation is not necessary, avoiding cases of difficult intubation or any trauma to the airways. An adequate informed consent and the appropriate compliance are elements of paramount importance in tailoring the anaesthetic strategy for pregnant women who need non-obstetric surgical management.

\section{Background}

Every year $2 \%$ of pregnant women undergo non-obstetric surgical interventions worldwide.'

According to the American College of Obstetricians and Gynaecologists Committee on Obstetric Practice, pregnant women should never be denied the most appropriate surgical treatment regardless of the trimester of pregnancy.

However, additional attention should be paid during the first trimester since there is the highest risk of inducing teratogenic mutations; similarly, during the third trimester, due to the possibility of preterm birth 
and low weight of the new-born great care should be paid.

It is well known that when a pregnant woman receives drugs, some of them cross the placenta reaching the foetal circulation through various mechanisms and may interact directly with the foetus.

For instance, there are several studies performed in rats, which have highlighted embryonic brain development after exposure to Sevoflurane; additionally, Propofol and Desflurane may also induce neuronal apoptosis resulting in cognitive dysfunction by inducing IL-6 production. '

On the other hand, the use of opioids during pregnancy, especially Remifentanil, is safer. This is mainly due to its peculiar pharmacokinetics, in fact it has a fast onset and offset. Therefore, it does not interfere with the physiological changes of pregnancy, and despite passing the placenta, it is quickly metabolized and prevents from using halogenated anaesthetic vapours such as Sevoflurane and Desflurane.'

Total Intravenous Anaesthesia (TIVA) is currently one of the preferred anaesthetic methods and it might be associated with the administration of alpha and/or beta blockers, halogenated or local anaesthetics.'

In literature there is a scantiness of data about the use of superficial sedation with or without analgesia in pregnant patients who need to undergo surgical interventions. '

For such reason it should be of paramount importance to avoid the use of inhaled anaesthetics which could bring about foetal damages, especially during the first trimester of pregnancy. In addition, this kind of approach may prevent from patient intubation, reducing possible traumatic events to the airways.

Reports concerning the use of analgosedation for head and neck surgery in pregnant women are extremely rare and the only cases described in literature are about eye and dental surgery.'

\section{Case Presentation}

We present the case of a 36-year-old woman during her 21st week of pregnancy, with a normal-sized foetus according to the gestational age on ultrasound exam and with no additional risk factors. The patient referred an increasing nasal obstruction associated with rhinorrhoea of the left nasal cavity. She also reported episodes of sleep apnoea and hyposmia.

The patient received a detailed otolaryngological examination which allowed to identify a mass within the left nasal cavity. The subsequent nasal endoscopy confirmed a greyish polypoid mass lesion with a multinodular surface occupying the entire left nasal fossa (Fig. 1). Further imaging studies included head

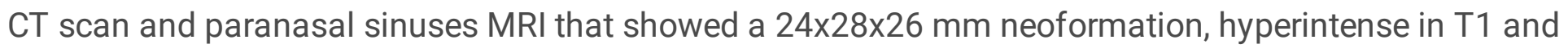
hypointense in $\mathrm{T} 2$, with a convoluted cerebriform pattern. The lesion totally obliterated the left maxillary sinus resulting in obstruction of the anterior osteo-meatal unit and ethmoidal sinusitis (Fig. 2). The adequate surgical treatment in such conditions should be a FESS with excision of the nasal lesion associated with left antrostomy and anteroposterior ethmoidectomy. 
The patient was therefore evaluated in a multidisciplinary setting and received a full gynaecological examination and foetal monitoring which did not reveal any anomalies.

In order to have a less invasive approach towards the foetus as possible the anaesthetist proposed an analgosedation with spontaneous breathing.

The patient consented to the surgical and anaesthetic procedure and signed a specific informed consent. She was transferred to Post Anaesthesia Care Unit (PACU) where she was placed a venous access with a $18 \mathrm{G}$ cannula needle into the back of her right hand.

The pregnant woman was carried on a stretcher to the operating room and placed in a supine position on the surgical bed with a wedge under the right side to facilitate venous return.

Vital parameters were monitored for the entire duration of the intervention. ECG monitoring using lead II showed sinus rhythm and heart rate (HR) was 80 beats per minute (BPM). Non-invasive blood pressure (NIBP) measured normal blood pressure (BP) $(120 / 80 \mathrm{mmHg})$. SpO2 was equal to $98-99 \%$ and respiratory rate was equal to 16 acts/minute. The patient was premedicated with $0.5 \mathrm{mg}$ of intravenous midazolam. After 5 minutes, in order to start analgosedation in association with surgical local anaesthesia, the target-controlled infusion was set with Remifentanil at $50 \mathrm{mcg} / \mathrm{ml}$ concentration and 1.5 $\mathrm{ng} / \mathrm{ml}$ dosage. The duration of the operation was 90 minutes and every 15 minutes a bolus of $0.5 \mathrm{mg}$ of Midazolam was administered to maintain sedation. The monitoring detected the following parameters during the operation: BP 110/60 mmHg, SpO2 99\%, FR 14 breaths per minute and HR 80 bpm. All parameters remained stable during all the time of surgery. The patient was kept in spontaneous breathing, ensuring valid airway protective reflexes - as cough, expiration reflex and swallowing reflexand making her promptly alert during all the time of surgical procedure.

After the surgical intervention, the patient was monitored in PACU. The clinical parameters were stable and comparable to the pre-operative monitoring. The gynaecological examination and foetal monitoring performed after the surgical procedure did not shown any kind of abnormalities.

The postoperative hospitalization lasted 24 hours and the patient did not present any complications.

Afterwards the patient was regularly assessed with clinical monitoring in the otolaryngology clinic. The final histological examination of the lesion turned out to be an inverted papilloma.

\section{Discussion And Conclusions}

Pregnant women undergo different physiological adaptations due not only to hormonal factors, but also to mechanical and metabolic elements resulting from the increase in size of the uterus and the growing foetal demands.

The respiratory system in particular faces major changes, including a $20 \%$ increase in oxygen consumption and a decrease in lung capacity. Such adaptations are responsible for the increased risk of 
hypoxemia related to the induction of general anaesthesia.

Another possible change reported in literature is the oedema of the oropharyngolaryngeal tissues; such oedema results into reduced glottic opening and difficulty in ventilation and tracheal intubation, starting from the second trimester of pregnancy. ${ }^{-}$

It is well known that when a pregnant woman receives drugs, some of them cross the placenta reaching the foetal circulation through various mechanisms and may interact directly with the foetus.

For instance, there are several studies performed in rats, which have highlighted embryonic brain development after exposure to Sevoflurane. Sevoflurane exposure at concentrations from $2-4 \%$ for 2 hours induces excessive levels of autophagy in the foetal brain through the activation of PTEN/Akt/mTOR pathway. ${ }^{5}$

Usually, foetal brain cells are characterized by a continuous turn-over: those cells which have accumulated damaged proteins and organelles undergo apoptosis through the PTEN transduction pathway. If the foetus is exposed to increased concentrations of Sevoflurane, the apoptotic pathway undergoes dysregulation due to the excessive activation of PTEN. The physiological cell proliferation is compromised with consequent neurotoxicity. Furthermore, Propofol and Desflurane can also induce neuronal apoptosis. ${ }^{6}$

On the contrary, administering opioids during pregnancy, especially Remifentanil, is safer because mainly due to its peculiar pharmacokinetics, in fact it is quickly metabolized avoiding interferences with physiological changes of pregnant and foetus as well. ${ }^{7-8}$

In literature the reported incidence of head and neck neoplasms during pregnancy is rare $(0.1 \%$ of all pregnancies) and oral cavity accounts for most cases.

Unfortunately, sometimes neoplastic lesions might be confused with rhinitis, a common disorder in pregnant women and frequently associated with a pre-existing asthmatic picture.

In this regard, typically Burkitt's lymphoma and dematiaceous fungal infections are more frequent especially among women who are immunocompromised or affected by aplastic anaemia.'

FESS represents the most common surgical procedure for treatment of nasal sinus diseases not responsive to drug therapy.

Total Intra-Venous Anaesthesia (TIVA) is currently one of the anaesthetic methods of choice, frequently associated with the administration of alpha and/or beta blockers, halogenated and local anaesthetics. ${ }^{9,10}$

The most common intraoperative occurrence during FESS, especially in the ethmoid and sphenoid sinus surgery, is represented by bleeding therefore maintaining normal blood pressure is of paramount 
importance. In fact, even minimal bleeding could compromise the visualization of the operating field, thus increasing the risk of iatrogenic lesions.'

In literature there are very few studies concerning the use of superficial sedation with or without analgesia in pregnant patients who need to undergo surgery for non-obstetrics indications. ${ }^{11,12}$

To the very best of our knowledge there are no available data about analgosedation and ENT surgery in pregnant women.

Interestingly, the use of superficial sedation maintains the physiological homeostasis of pregnant woman with improvements both for perfusion and oxygenation. In fact, general anaesthesia can affect uterine blood flow both for alterations in perfusion pressure and for variations in vascular resistance. Furthermore, maintaining spontaneous ventilation can prevent oxygenation defects that could lead to an increased risk of foetal asphyxia.

A key point which needs to be clearly defined is the correct choice of the patient for patient's compliance is essential; in fact, the pregnant woman has to be able to precisely follow the surgeons and anaesthetists' instructions during the operation.

For such reason, it is crucial to explain in detail the anaesthetic techniques that is going to be used, specifically focusing on risks and benefits thus motivating the patient.

\section{Abbreviations}

\section{FESS}

Functional Endoscopic Sinus Surgery

TIVA

Total Intravenous Anaesthesia

CT

Computed Tomography

MRI

Magnetic Resonance Imaging

PACU

Post Anaesthesia Care Unit

ECG

Electrocardiography

$\mathrm{HR}$

Heart Rate

BPM

Beats Per Minute

NIBP

Non-Invasive Blood Pressure 
BP

Blood Pressure

$\mathrm{SpO} 2$

peripheral oxygen saturation

\section{Declarations}

Ethics approval and consent to participate: Ethics approval was waived because our treatment was conducted according to good clinical practice.

Consent for publication: Written informed consent was obtained from the patient for publication of this Case report and any accompanying images. A copy of the written consent is available for review by the Editor of this journal.

\section{Availability of data and materials:}

Data used during the current study is available from the corresponding author on reasonable request.

Competing interests: Authors declare they have no competing interests.

Funding: None

\section{Authors' contributions:}

CM, SM examined patient and performed anaesthesia and surgery.

$\mathrm{FM}, \mathrm{AL}, \mathrm{AC}$ and $\mathrm{FI}$ were major contributors in writing the manuscript.

GB and LT gave final approval to the manuscript.

All authors read and approved the final manuscript.

Acknowledgements: None

\section{References}

1. Balinskaite V, Bottle A, Sodhi V, Rivers A, Bennett PR, Brett SJ, et al. The Risk of Adverse Pregnancy Outcomes Following Nonobstetric Surgery During Pregnancy: Estimates From a Retrospective Cohort Study of 6.5 Million Pregnancies. Ann Surg. 2017;266(2):260-266.

2. Van De Velde M, De Buck F. Anesthesia for non-obstetric surgery in the pregnant patient. Minerva Anestesiol. 2007;73(4):235-40.

3. ACOG Committee Opinion No. 775: Nonobstetric Surgery During Pregnancy. Obstet Gynecol. 2019;133(4):e285-e286. 
4. Cho S, Chung RK, Jin SH. Factors Affecting Maternal and Fetal Outcomes of Non-Obstetric Surgery and Anesthesia during Pregnancy: a Retrospective Review of Data at a Single Tertiary University Hospital. J Korean Med Sci. 2020;35(16):e113.

5. Li X, Wu Z, Zhang Y, Xu Y, Han G, Zhao P. Activation of Autophagy Contributes to Sevoflurane-Induced Neurotoxicity in Fetal Rats. Front Mol Neurosci. 2017;10:432.

6. Hirotsu A, Iwata Y, Tatsumi K, Miyai Y, Matsuyama T, Tanaka T. Maternal exposure to volatile anesthetics induces IL-6 in fetal brains and affects neuronal development. Eur J Pharmacol. 2019;863:172682.

7. Kan RE, Hughes SC, Rosen MA, Kessin C, Preston PG, Lobo EP. Intravenous remifentanil: placental transfer, maternal and neonatal effects. Anesthesiology. 1998;88(6):1467-74.

8. Marsh BJ, Sinskey J, Whitlock EL, Ferschl MB, Rollins MD. Use of Remifentanil for Open in utero Fetal Myelomeningocele Repair Maintains Uterine Relaxation with Reduced Volatile Anesthetic Concentration. Fetal Diagn Ther. 2020;47(11):810-816.

9. Ragab SM, Hassanin MZ. Optimizing the surgical field in pediatric functional endoscopic sinus surgery: a new evidence-based approach. Otolaryngol Head Neck Surg. 2010;142(1):48-54.

10. Hamed MA. Comparative Study between Magnesium Sulfate and Lidocaine for Controlled Hypotension during Functional Endoscopic Sinus Surgery: A Randomized Controlled Study. Anesth Essays Res. 2018;12(3):715-718.

11. Neuman G, Koren G. Safety of procedural sedation in pregnancy. J Obstet Gynaecol Can. 2013;35(2):168-173.

12. Bonnet MP. Sedation and anaesthesia for non-obstetric surgery. Anaesth Crit Care Pain Med. 2016;35 Suppl 1:S35-S41.

13. Peeler CE, Villani CM, Fiorello MG, Lee HJ, Subramanian ML. Oral versus Intravenous Sedation Study Group. Patient Satisfaction with Oral versus Intravenous Sedation for Cataract Surgery: A Randomized Clinical Trial. Ophthalmology. 2019;126(9):1212-1218.

14. Flanagan D. Oral triazolam sedation in implant dentistry. J Oral Implantol. 2004;30(2):93-7.

15. Marangoni F, Cetin I, Verduci E, Canzone G, Giovannini M, Scollo P, et al. Maternal Diet and Nutrient Requirements in Pregnancy and Breastfeeding. An Italian Consensus Document. Nutrients. 2016;8(10):629.

16. Soares MJ, Iqbal K, Kozai K. Hypoxia and Placental Development. Birth Defects Res. 2017;109(17):1309-1329.

17. Rocke DA, Murray WB, Rout CC, Gouws E. Relative risk analysis of factors associated with difficult intubation in obstetric anesthesia. Anesthesiology. 1992;77(1):67-73.

18. Pilkington S, Carli F, Dakin MJ, Romney M, De Witt KA, Doré CJ, et al. Increase in Mallampati score during pregnancy. Br J Anaesth. 1995;74(6):638-42.

19. Le Guevelou J, Lebars S, Kammerer E, de Gabory L, Vergez S, Janot F, et al. Head and neck cancer during pregnancy. Head Neck. 2019;41(10):3719-3732. 
20. Powell H, Murphy VE, Hensley MJ, Giles W, Clifton VL, Gibson PG. Rhinitis in pregnant women with asthma is associated with poorer asthma control and quality of life. J Asthma. 2015;52(10):1023-30.

21. Yoruk O, Ucuncu H, Gursan N, Aktan B, Sutbeyaz Y, Altas E, et al. Sinonasal Burkitt lymphoma presenting as a nasal polyposis in a pregnant woman. J Craniofac Surg. 2009;20(4):1059-60.

22. Derber $C$, Elam K, Bearman G. Invasive sinonasal disease due to dematiaceous fungi in immunocompromised individuals: case report and review of the literature. Int J Infect Dis. 2010;14 Suppl 3:e329-32.

23. Weber RK, Hosemann W. Comprehensive review on endonasal endoscopic sinus surgery. GMS Curr Top Otorhinolaryngol Head Neck Surg. 2015;14:Doc08.

24. Sieśkiewicz A, Reszeć J, Piszczatowski B, Olszewska E, Klimiuk PA, Chyczewski L, et al. Intraoperative bleeding during endoscopic sinus surgery and microvascular density of the nasal mucosa. Adv Med Sci. 2014;59(1):132-5.

25. Parvizi A, Haddadi S, Faghih Habibi A, Nemati S, Akhtar N, Ramezani H. Dexmedetomidine Efficacy in Quality of Surgical Field During Endoscopic Sinus Surgery. Iran J Otorhinolaryngol. 2019;31(106):281-288.

\section{Figures}




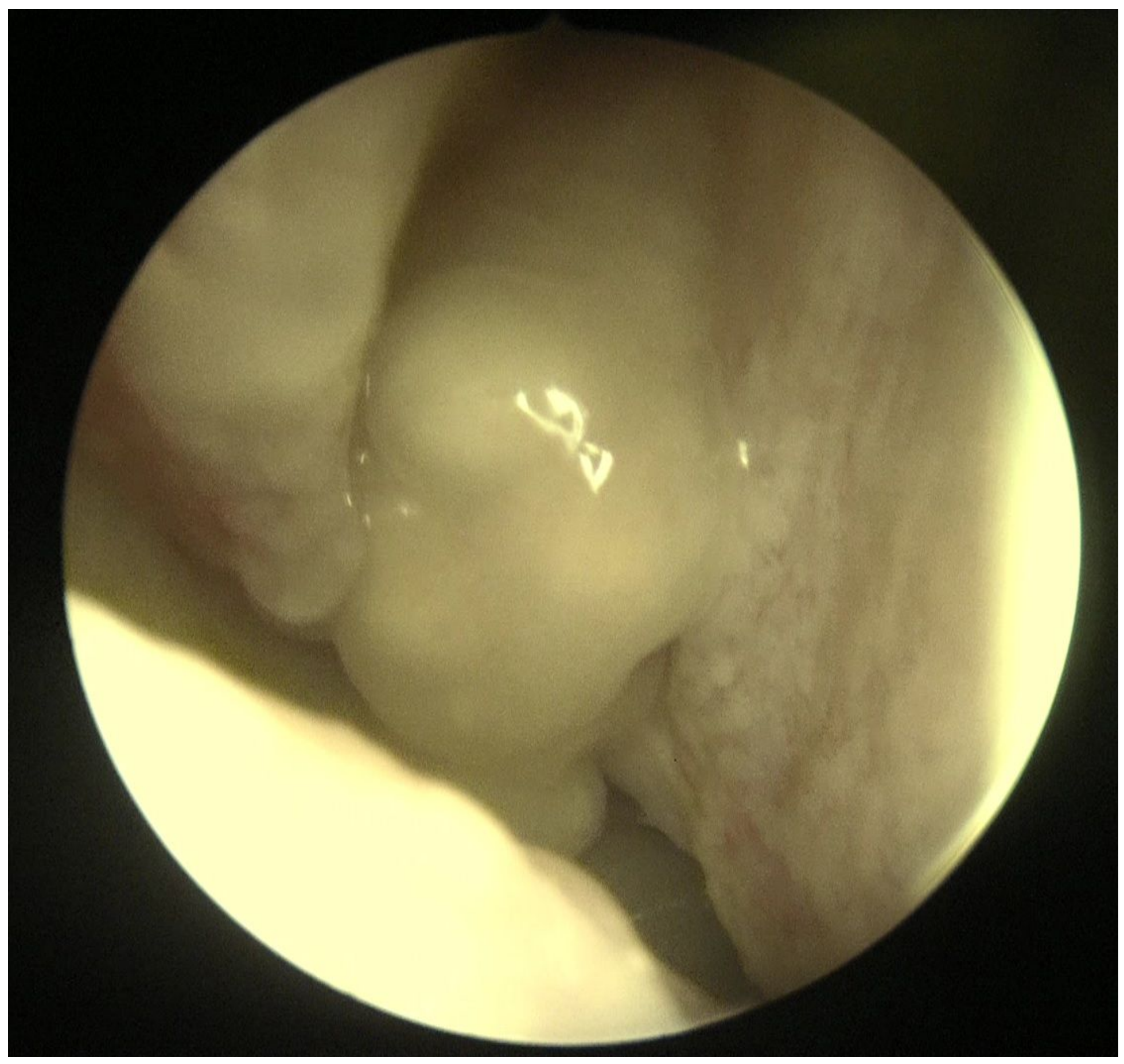

\section{Figure 1}

Endoscopic appearance of a greyish polypoid mass lesion with a multinodular surface occupying the entire left nasal fossa. 

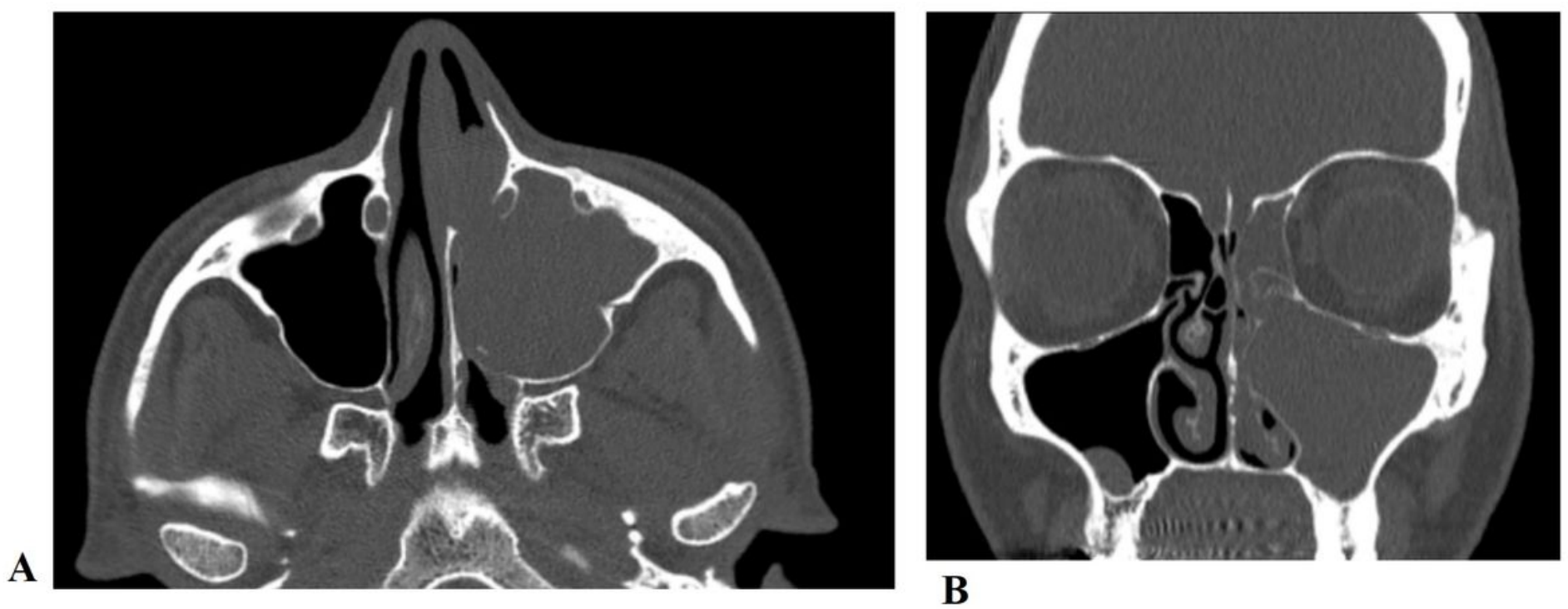

Figure 2

Head CT scan, 2a- Axial view, 2b- Coronal view: Evidence of a lesion, 24x28x26 mm in size, totally obliterating the left maxillary sinus resulting in obstruction of the anterior osteo-meatal unit and ethmoidal sinusitis. 